\title{
The use of thermography in the diagnosis of ship piston internal combustion engines
}

\author{
Jan Monieta ${ }^{1, * *}$ \\ ${ }^{1}$ EDP, Maritime University of Szczecin, Wały Chrobrego 1-2, 70-500 Szczecin, Poland
}

\begin{abstract}
The intensity of infrared radiation emitted by objects depends mainly on their temperature. One of the diagnostic signals may be the temperature field. In infrared thermography, this quantity is used as an indicator of the technical condition of marine objects. The article presents an overview of the use of infrared thermography for the diagnosis mainly of marine piston floating objects and various types of reciprocating internal combustion engines as well as examples of own research results. A general introduction to infrared thermography and common procedures for temperature measurement and non-destructive testing are presented. Experimental research was carried out both in laboratory conditions and in the operating conditions of sea-going vessels. Experimental studies consisted of the presentation of photographs of the same objects made in visible light and the use of infrared thermography. The same objects were also compared, but for different cylinders of the tested internal combustion engines as well as for the up state and fault state. The characteristics of the temperature values at selected points were taken depending on the engine load along with the approximation mathematical models of these dependencies.
\end{abstract}

\section{Introduction}

Infrared thermography is a technique for producing an image of the invisible to eyes infrared light emitted by objects due to their thermal condition. A typical a thermography camera produces a live video picture of heat radiation intensities. More sophisticated cameras can actually measure the temperature values of any object or surface in the fieldof-view and produce colour images that make interpretation of thermal patterns easier. An image produced by an infrared (IR) camera is called a thermogram [1].

The properties of measuring heat transmission from objects using the thermography camera are more than an interesting novelty. An advance in IR sensor production has helped the infrared viewing technology to be adopted as a cost-effective, non-invasive measuring method. Advanced optics and sophisticated software interfaces continue to add to the variety of Thermal IR cameras.

However, the most powerful means of thermal energy transfer is radiation, which moves at the speed of light. Infrared energy is emitted by all materials above $0 \mathrm{~K}$. Infrared radiation is part of the electromagnetic spectrum and occupies frequencies between visible light and radio waves. The infrared part of the spectrum spans wavelengths from $0.7 \mu \mathrm{m}$ to $1000 \mu \mathrm{m}$ [1]. Within this wave band, only frequencies of $0.7 \mu \mathrm{m}$ to $20 \mu \mathrm{m}$ are used for practical, everyday temperature measurement.

The three methods by which heat flows from one object to another are: radiation, convection and conduction. These three phenomena in most situations they occur together. The invention of the thermal imaging camera has caused a number of changes in the field of temperature measurement. The measurement method bases its operation on the phenomena of the objects' impact relative to their surrounding space. The use of

${ }^{*}$ Corresponding author: j.monieta@am.szczecin.pl 
electromagnetic radiation reception in the low energy range of this radiation has contributed to the development of new directions in exact sciences.

\section{An examples of the use of thermography in the diagnostics of marine combustion engines}

The intensity of the infrared radiation emitted by objects is mainly a function of their temperature [2]. In infrared thermography, this feature is used for multiple purposes: as a health indicator in medicine, process monitoring, manufacturing inspection, and nondestructive testing, as a sign of malfunction in mechanical and electrical maintenance or as the indicator of heat loss in objects. In paper presents a review of infrared thermography focused on two applications [3]: temperature measurement and non-destructive testing for the general introduction to infrared thermography and the common procedures for temperature measurement and non-destructive testing are presented.

A ship classification and certification body Lloyd's Register, made the prediction, that the near future, mechanical machinery onboard vessels will also benefit from thermal imaging to repaired, especially as a pre-docking strategy to identify and target equipment and systems which need maintenance as well as to eliminate necessary works [1]. Commercial ships are objects for thermographic inspections with big machinery, vast installations, extended electronic systems that can hardly be surveyed by visual inspections. According to the International Convention for the Safety of Life at Sea (SOLAS), the maximum surface temperature of machinery, parts and components in a vessel's engine room should not rise above $220^{\circ} \mathrm{C}$ [1]. The majority of engine room fires are caused by ruptured fuel or oil pipes which eventually spray fuel or oil on adjacent hot surfaces [1, 4]. IR thermography has the advantage of being a noncontact inspection and measurement tool able to display and store exact temperature values as well as visual evidence. When something happens to the engines or to other vital installations, inspection tool as an infrared camera can be used. Thermography is used in marine applications for engineering inspection of electrical and mechanical systems of ships. IR thermography can play a major role in maintaining the safety and reliability of marine floating objects where catastrophic failure could lead to the loss of a vessel or its crew.

The thermal imaging camera has the receiving function here and is a signal transducer. Advanced thermal imaging cameras are characterized by high resolution of the image being processed and recorded the image on a storage medium. Saving images or video recordings can then be played back to analyse the course of changes in temperature areas [5]. Thermal imaging cameras have recently been the object of interest of many specialists dealing with diagnostics [5-7].

In the modern internal combustion engines it strives to reduce the toxicity of exhaust gases, fuel economy and how best cohesive properties. In the operation of the engine of internal combustion important element is the ongoing control of its technical state. The essence of activity, directed is on the maximum use of the time of the engine and its diagnosis. This makes it possible to diagnose the consumption of failure. There are many methods of diagnostics. One of them is the thermography diagnosis for maintenance. In the article presents technical state assessment of selected components of the engine of internal combustion of crank train and oil supply system based on the obtained IR image [6].

Recently, thermography has proved to be a powerful tool to deepen the knowledge about the chemical and physical processes that take place in automotive engine. $2 \mathrm{D}$ infrared (IR) chemiluminescence measurements have been performed in an optical Common Rail self-ignition engine in order to investigate the different processes that regulate the pollutant emission formation [8]. The time evolution of the injected fuel, of the flames, and of the burned gases has been analyzed. Infrared images have been detected in the spectrum from $1.5 \mathrm{mu} \mathrm{m}$ to $5 \mathrm{mu} \mathrm{m}$. It has allowed obtaining more information with respect to the visible 
imaging and it has been possible to detect the fuel vapour and the burned gas motion in the combustion chamber for a longer period with respect to visible range. $\mathrm{CO}_{2}$ evolution in the available volume during several combustion phases has been detected: during the inlet phase, during the combustion phase, in the burned gases cloud that moves toward the outlet valves. The results obtained suggest IR diagnostics as useful tool for engine control.

Infrared thermography has become the powerful tool for basic and applied scientific research and for the application in maritime affairs. As a prognosis maintenance tool [9], IR thermography has the ability to identify problems before they occur. It is especially helpful for trouble shooting potential electrical overloads, worn or bad circuit breakers. IR thermography can also be used to detect bad bearings of shafts or any application where heat detection would be beneficial. This paper has basic principles of IR thermography are presented and examples of the tool application in maritime affairs are given [1].

One of the benefits of using thermal imaging is the ability to predict possible the fire hazards due to increases in temperature in controlled areas in a particular marine object. The work proposes the possibility of applying new electronic and computer technology as part of the ship's fire detection system, such as the use of computer vision, using existing marine thermography systems and installing thermal imaging IR cameras on the same system [5]. There has been proposed communication between the thermal imaging system and the fire detection and central alarm system of the ship. The visual analysis of certain areas on board the ship and the related facilities inside it, with the addition of certain software applications into the existing video technology system, makes is the fire alarm system. With the forecast and send early warnings to the ship's central fire alarm system, thereby contributing to improved safety with to equipment, the ship as a whole, cargo and human lives. The spreads widely very fastly in high temperatures and becomes the cause of great damage to ship equipment [10], often resulting in the loss of the ship. Videotapes from thermal cameras can be used for risk analysis and the prevention of future ship fire incidents. It is possible the fast detection of temperature changes very early warning.

The article presented non destruction method and results of using thermal method in diagnostic of marine combustion engines operation of fishing fleet vessel [7]. The article presents a mathematical model for determining the temperature distribution by means of thermovision [5]. Knowledge of this model makes it possible to analyse the influence of external factors [11] on the accuracy of temperature determination by thermovision.

\section{Principles of Infrared Thermography}

The basic dependence used to determine the temperature is the thermovision method is basic equation of radiation. Effective radiation power of the thermographic camera that reaches the lens from the surface of the object being examined consists of the following components [12]:

- the effective radiation power emitted by the tested object,

- radiation of the background reflected from the object,

- radiation of your own atmosphere.

The hotter an object becomes the more infrared energy it emits. The Stefan-Boltzmann law, states that the total energy radiated per unit surface area of a black body in unit time $E_{B B}$ is directly proportional to the fourth power of the black body absolute temperature $T$ :

$$
E_{B B}=\sigma \cdot T^{4}\left[\mathrm{~W} / \mathrm{m}^{2}\right]
$$

The constant of proportionality $\sigma$, called the Stefan-Boltzmann constant is nonfundamental in the sense that it derives from other known constants of nature. 
When the object is at thermal equilibrium, the amount of absorption will equal the amount of emission. The Kirchoff's law determined for any material, the emissivity and absorptance of the body are equal at any specified temperature and wavelength [3]:

$$
\varepsilon_{\lambda}=a_{\lambda}
$$

where:

$a$ - coefficient of absorptance,

$\lambda$-wavelength.

When the infrared energy radiated by real object reaches another body, a portion of the energy received will be absorbed $a$, a portion will be reflected $r$ and, if the body is not opaque, a portion $t_{r}$ will be transmitted. The sum of the individual parts is added up to the initial value of radiation which left the source:

$$
a+r+t_{r}=1
$$

The total radiation received by the camera $W_{t}$ comes from three sources: the emission of the target objects $E_{o}$, the emission of the surroundings and reflected by the object $E_{r}$ and the emission of the atmosphere $E_{a}$. It can be expressed as equation:

$$
W_{t o}=E_{o}+E_{r}+E_{a}
$$

The total strength of the radiation can be expressed with the formula FLIR Systems:

$$
W_{\mathrm{to}}=\varepsilon_{o} \tau_{o} W_{\mathrm{o}}+\left(1-\varepsilon_{o}\right) \tau W_{\mathrm{r}}+\left(1-\tau_{a}\right) W_{\mathrm{a}}
$$

where:

$\varepsilon_{o} \tau_{o} W_{\mathrm{o}}$ - is the emission of radiation from the object,

$\varepsilon_{o}$ - the emission of the object,

$\tau_{a}$ - the atmospheric release,

$\left(1-\varepsilon_{o}\right) \tau W_{r}-$ the reflection from the environment,

$\left(1-\varepsilon_{o}\right)$ - the reflection of the object,

$\tau_{a} W_{r}$ - the temperature of the environment;

$\left(1-\tau_{\mathrm{a}}\right) W_{\mathrm{a}}$ - the emission of radiation from the atmosphere, $\left(1-\tau_{\mathrm{a}}\right)$ is the emission of the atmosphere and $W_{\mathrm{a}}$ is the temperature of the atmosphere.

The basic equation of radiation for the complexity of the measurement scene can be saved:

$$
W_{t o}=\tau_{a} \varepsilon_{o} \sigma T_{o}^{4}+\tau_{a}\left(1-\varepsilon_{o}\right) \sigma T_{r}^{4}+\left(1-\tau_{a}\right) \sigma T_{a}^{4}[\mathrm{~W}]
$$

Hence the measured temperature of object is equal:

$$
T_{o}=\sqrt[4]{\frac{W_{t o}-\left(1-\varepsilon_{o}\right) \tau_{a} \sigma T_{r}^{4}+\left(1-\tau_{a}\right) \sigma\left(1-\tau_{a}\right) \sigma T_{a}^{4}}{\varepsilon_{o} T_{a} \sigma}}
$$

In order to solve equation (6), the following parameters must be supplied: the emissivity of the object, the reflected temperature $T_{r}$, the transmittance of the atmosphere and the temperature of the atmosphere $T_{a}$. The transmittance of the atmosphere is estimated using the distance from the object to the camera and the relative humidity. This value is very close to one. The temperature of the atmosphere is obtained using a common thermometer. However, as the emittance of the atmosphere is very close to zero $(1=a)$, this parameter has little influence on the temperature measurement. The emissivity of the object and the reflected temperature has but the very high influence on the temperature measurement. 


\section{Methodology and research objects}

The object of thermal imaging studies were the combustion engines driving generators of 3 types and one main drive. The research was carried out both in the laboratory conditions of the Maritime University of Szczecin and in the conditions of operation of sea-going vessels. Different types of FIR cameras were used for the tests. First, the measuring track was tested by comparing the temperature readings with thermometers and the thermovision camera. Thermal imaging was preceded by the configuration of the camera settings to the conditions that prevailed during the tests, which is why the parameters of the reference conditions were measured: ambient temperature, atmospheric pressure and relative humidity.

\section{Sample investigations results}

The measurement path was calibrated by measuring the temperature with contactless and contactless devices inside the muffle furnace for calibrating thermoelectric thermometers and a thermal imaging camera (Fig. 1). The same tests were carried out in the conditions of sea-going vessels. Differences in indications were insignificant.

a)

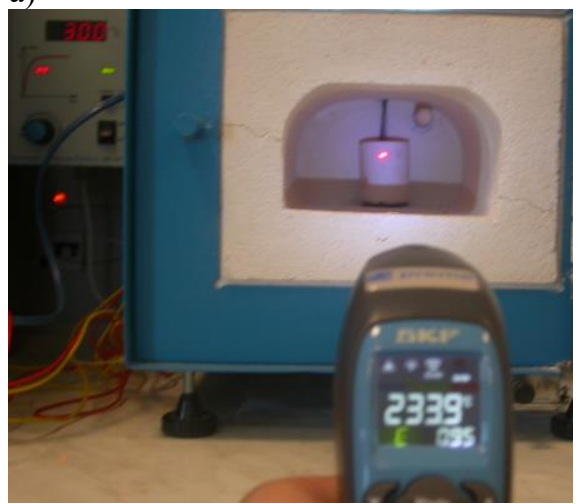

b)

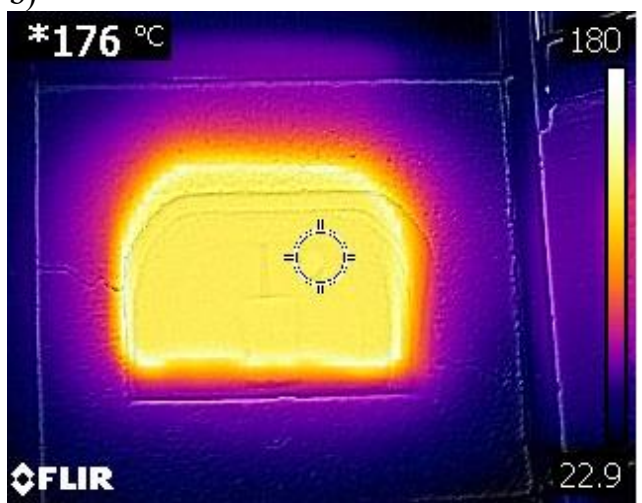

Fig. 1. View of a muffle furnace for calibrating thermometers with measurement using a contactless thermometer a) and a photo taken with a thermal imaging camera b).

Fig. 2 presents a global view of a functioning engine driving the generator in the compartment of the ship's power plant and the temperature field from the infrared camera. Fig. 3, 4 and 5 show images in visible light and infrared from the thermovision camera of such elements as an injection pump, body of injector valve and head cover together with the temperature values.

a)

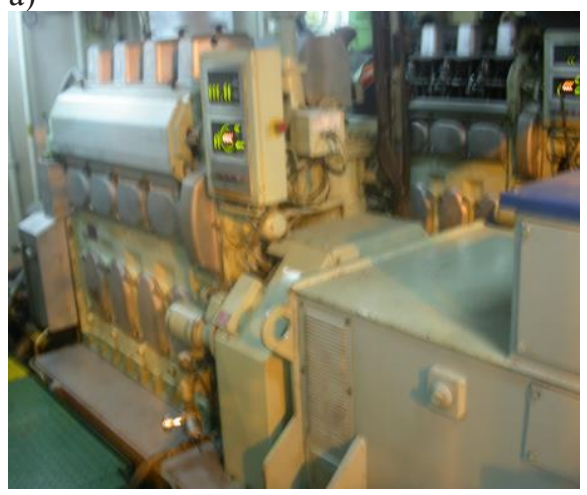

b)

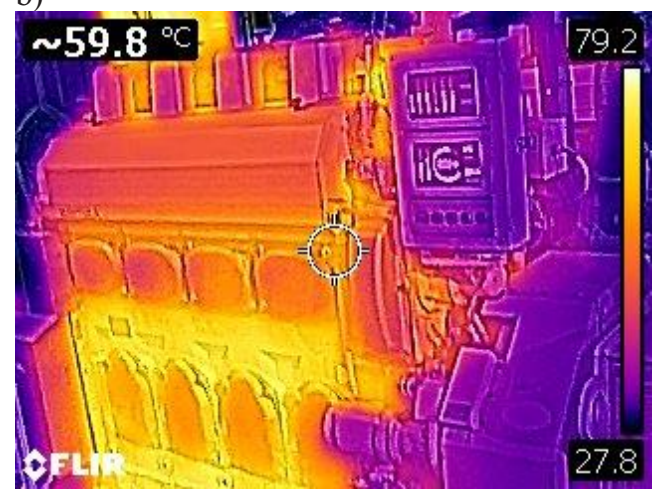

Fig. 2. Photograph in visible light of the engine with the generator (a) and thermographic image b). 
a)

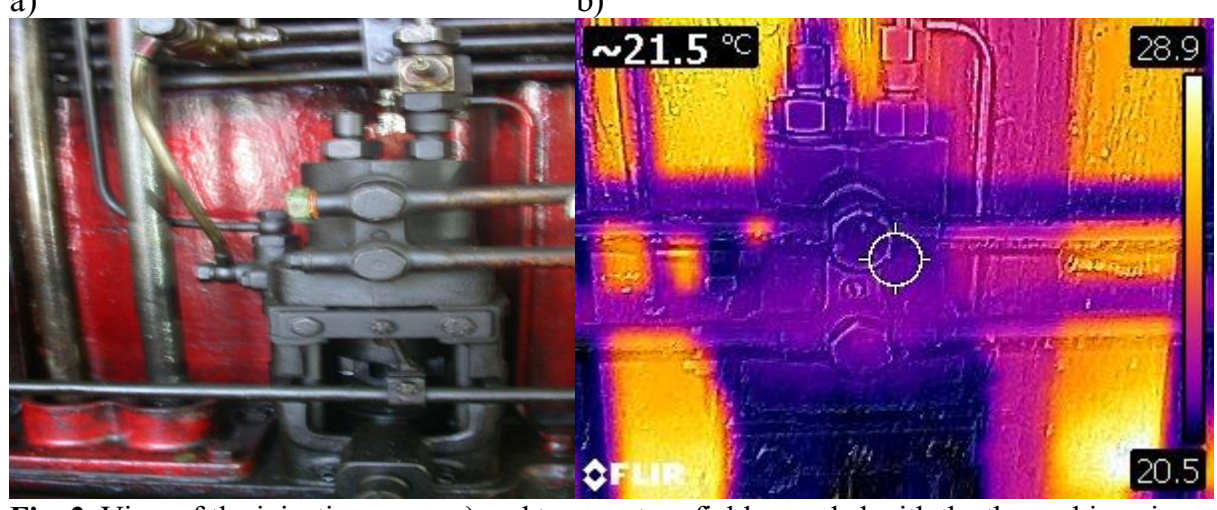

Fig. 3. View of the injection pump a) and temperature field recorded with the thermal imaging camera b).

a)

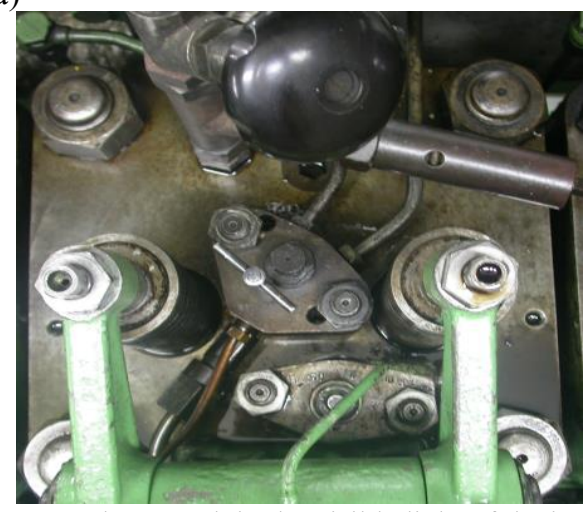

b)

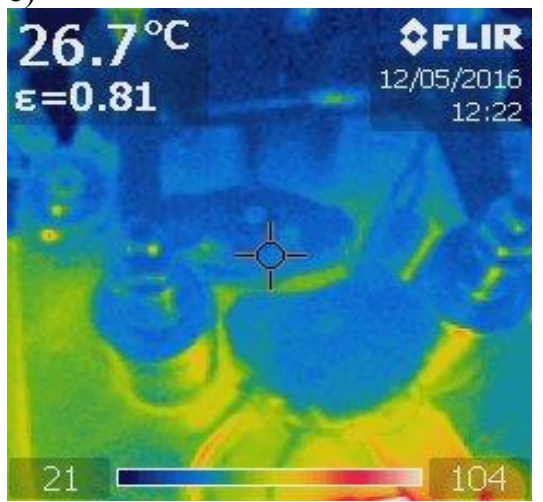

Fig. 4. Photograph in the visible light of the internal combustion engine head without cover a) and the temperature field from the infrared camera.

a)

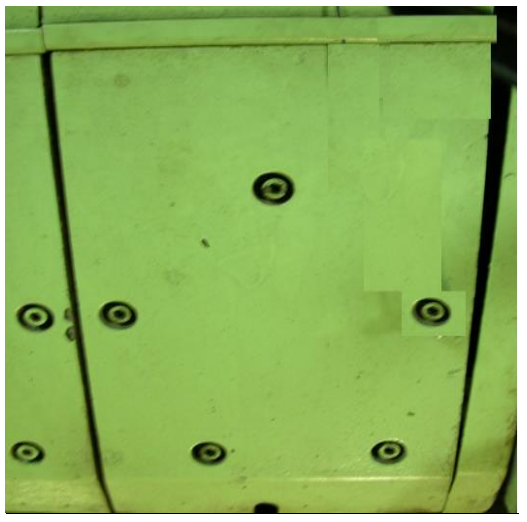

b)

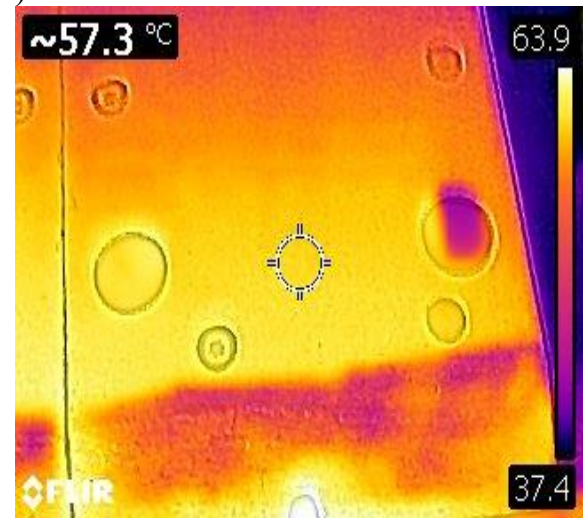

Fig. 5. View of the head cover a) and the temperature field recorded with the thermal imaging camera b).

The tests were carried out for various cylinders of combustion engines, with different loads and for different technical conditions. In contrast, Fig. 6 shows dependencies the 
temperature of the injector bodies depending on the engine load. The curve for the third cylinder deviating from the others results from the inability state of the injection subsystem.

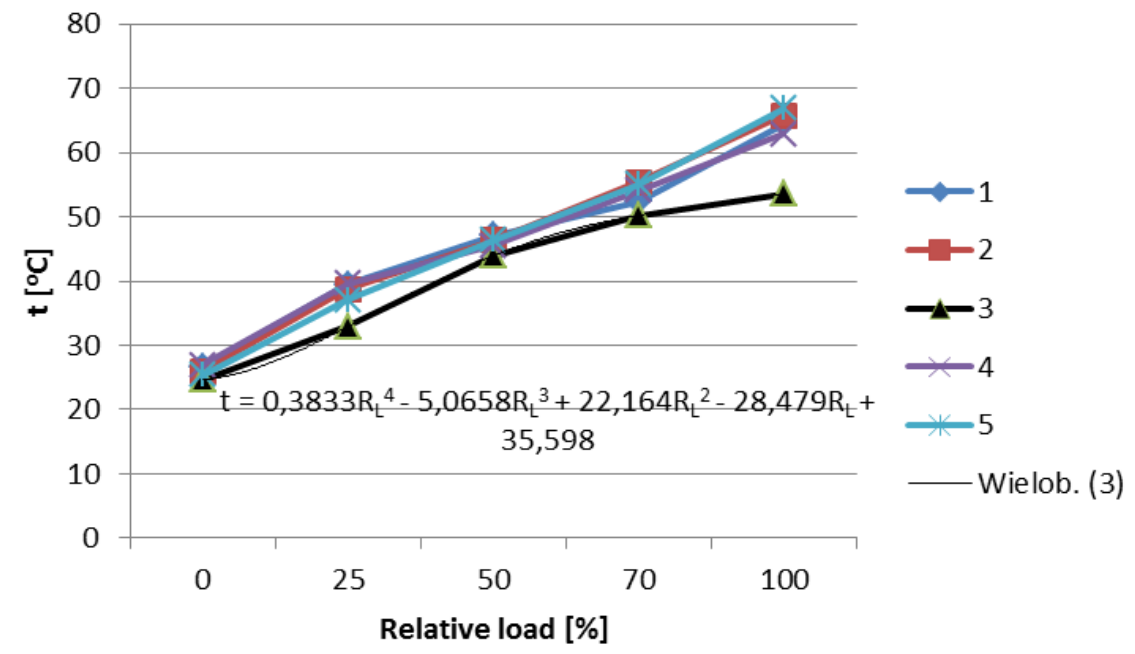

Fig. 6. The influence of engine load on temperature values on the injector body for five cylinders of an internal combustion engine.

This technique is based on the polynomial fit of each pixel time history from the given thermographic image sequence. The evolution of the temperature is adjusted to an $n$ degree polynomial for input value $x$, what described in equation [13]:

$$
T(t)=a_{n} x^{n}+a_{n-l} x^{n-1}+\ldots+a_{1} x+a_{0}
$$

This paper presents an example of the polynomial model for the temperature dependence of the injector body function on the relative load $R_{L}$ of the combustion engine for one of the curves of Fig. 6.

$$
t=0,3833 R_{L}{ }^{4}-5,0658 R_{L}{ }^{3}+22,164 R_{L}^{2}-28,479 R_{L}+35,598
$$

\section{Conclusions}

Diagnostics of ship engine facilities using thermovision and thermography are noninvasive, which is likely to be recognized by classification societies. Application is possible for objects with different temperature fields. The measurement process itself takes place without any prior preparation and quickly.

The benefits of using thermovision imaging are the ability to predict possible fire hazards caused by growth of temperature and catastrophic failures in controlled areas in specific marine objects or systems.

The article presents the results of preliminary experimental research. Photographs in visible light and images from infrared camera of the surface elements of marine engines were placed.

Further research should demonstrate the usefulness of selected diagnostic symptoms, check the possibility of detecting specific damages, determine the measurement errors and combine the monitoring with the alarm system of the ship's actuator.

The results so far have to be considered as encouraging.

\section{References}

1. V. Pašagić, M. Mužević, D. Kelenc, Brodogradnja 59, 2, pp. 123-130 (2008) 
2. C. Meola, Origin and Theory of Infrared Thermography. In Infrared Thermography: Recent Advances and Future Trends; Meola, C., Ed.; Bentham Science: New York, NY, USA (2012).

3. R. Usamentiaga, P. Venegas, J. Guerediaga, L. Vega, J. Molleda and F. G. Bulnes, Sensors, 14, pp. 12305-12348 (2014)

4. J. Monieta, Scient. Journ. of the Maritim. Univ. of Szczecin, 47, 119, pp. 36-41 (2016)

5. M. Bistrović, P. Ristov, D. Komorčec, Scient. Journ. of the Marit. Univ. of Szczecin, 50, 122, pp. 23-29 (2017)

6. P. Jarmużek, W. Sawczuk, Log. 3, pp. 2557-2562 (2014)

7. O. Klyus, C. Behrend, P. Rajewski, M. Szczepanek, O. Bezyukov, A. Kardanov, Scient. Journ. of the Maritim. Univ. of Szczecin, 35, 107, pp. 36-41 (2013)

8. E. Mancaruso, L. Sequino, M.B Vaglieco, 16th Fot. AEIT Italian Conf. on Phot. Technol. Loc., Naples, Italy, 12-14 (2014)

9. B. Żóltowski, C. Cempel, Engineering of diagnostics machines (in Polish), Polish Society of Technical Diagnostics, Warsaw, Bydgoszcz, Radom (2004).

10. J. Monieta, Tribol. 4, pp. 55-63 (2017)

11. C. Jermak, R. Majchrowski, J. Monieta, Combust. Eng. 146, 3, pp. 71-72, cd. pp. 1-8 (2011)

12. Z. Rymarczyk, M. Dzierzgowski, M. Strzeszewski, The use of thermography for a qualitative assessment of thermal protection of buildings in: "Energy-efficient shaping of the internal environment" (work done by Z. Popiołek). (in Polish), Silesian University of Technology, Gliwice (2005)

13. A.V. Nguyen, N.J. Cohen, H. Lipman, C.M. Brown, N.A. Molinari, W.L Jackson, H. Kirking, P. Szymanowski, T.W. Wilson, B.A. Salhi, et al, Infect. Dis., 16, pp. 17101717 (2010).

\section{Acknowledgements}

This research outcome has been achieved under the research project "Ecological and economic aspects of the operation of selected elements of marine power systems" No. 1/S/IESO/2014 financed from a subsidy of the Ministry of Science and Higher Education for statutory activities. 